\title{
Zu genau hingesehen
}

_ Eine 36-jährige Frau litt seit etwa drei Wochen unter Juckreiz der Augenlider. Ihren Ehemann juckte es dagegen in der Schamregion. Bei der Lupenuntersuchung der Augenlider erkannte man einige wenige Läuse und Nissen an der Basis der Wimpern (Abb. 1).

Am rechten Auge bestand eine leichte konjunktivale Injektion, ansonsten war das Auge unauffällig. Im Bereich des Schamhaares der Frau fanden sich ebenfalls multiple Läuse und Nissen. Andere behaarte Regionen wie Augenbrauen, Achselhaar, Körperbehaarung oder Kopfhaut waren nicht befallen. Mikroskopisch erkannte man Phthirus pubis, vulgo Filzläuse, mit den dazu gehörigen Nissen. Ein Screening auf andere sexuell übertragbare Erkrankungen wie HIV, Syphilis, Hepatitis $\mathrm{B}$ und $\mathrm{C}$ erwies sich als unauffällig.

Die Läuse wurden mit einer Pinzette von den Wimpern entfernt und die Wimpern zweimal täglich mit Vaseline bestrichen. Die Haare der Schamregion wurden abrasiert und die Haut mit 10\%-iger Schwe- felsalbe einmal täglich über drei Tage hinweg behandelt. Die Patientin wurde angewiesen, alle Kleidungsstücke und sonstige Infektionsträger zu waschen. Ihr Gatte wurde in gleicher Weise behandelt. Zwei Wochen später hatte sich der Juckreiz bei beiden Personen zurückgebildet, Augenlider und Schamhaarbereich waren frei von Parasiten.

\section{Kommentar}

Obwohl Phthirus pubis praktisch alle behaarten Stellen befallen kann, ist die Manifestation an den Augenlidern bei gesunden Erwachsenen doch extrem selten. In diesem Fall würde wohl die detaillierte Anamnese weiter helfen. Nachdem die Infektion bei den Erwachsenen in der Regel sexuell übertragen wird, empfiehlt sich ein Screening nach weiteren sexuell übertragbaren Erkrankungen. Bei Kindern ist die Manifestation gerade an den Augenlidern aber besonders häufig. Vaseline ist sehr effektiv bei der Eradikation von
Läusen, zerstört aber nicht die Eier. Sieben Tage nach Beginn der therapeutischen Maßnahme sollten die Haare erneut auf das Vorliegen von Filzläusen untersucht werden. Finden sich weitere Läuse, so ist ein anderes therapeutisches Regime anzuwenden, zum Beispiel Lindan, Permethrin, Malathion oder Phenothrin. Kleidung, Handtücher und Bettzeug sollten mit einer Temperatur von mindestens $55{ }^{\circ} \mathrm{C}$ gewaschen werden.

H. S. FüEßL =
- D.L. Ma und S. Vano-Galvan

(Korr.: Department of Dermatology, Peking Union Medical College Hospital, Beijing, China) Infestation of the eyelashes with Phthirus pubis. CMAJ 2010; doi:10.1503/ cmaj.090793

\section{Müdigkeit ist auch hörbar}

\author{
Wissenschaftler der University of \\ Melbourne (Australien) haben \\ herausgefunden, dass Erschöpfung \\ den Klang der Stimme beeinflusst. \\ Nach einer längeren Phase ohne \\ Schlaf verändert sich die Stimme. Sie \\ wird langsamer, unkontrollierter und \\ höher.
}

- 18 Jugendliche nahmen an Sprachtests teil. Über 24 Stunden mussten sie alle zwei Stunden einen gedehnten Ton von sich geben, einen Text lesen und zählen. Schlafen durften sie zwischen diesen Tests nicht.
Im Verlaufe von 24 Stunden verlangsamte sich die Sprechgeschwindigkeit zunehmend. Die Schwankungen in der Tonhöhe nahmen zu. Die tiefen Klänge gingen verloren. Je länger der Mensch wach ist, umso mehr verliert er die Feinkontrolle über die Stimmbandmuskulatur.

\section{Kommentar}

Die Wissenschaftler hoffen auf ein simples Verfahren, um rechtzeitig über eine Stimmanalyse vor Übermüdung zu warnen und gefährliche Situationen zu verhindern. Schichtarbeiter, Soldaten, Mediziner oder
Fernfahrer könnten von einem entsprechendem Warnsystem profitieren.

K. MALBERG

- A.P. Vogel et al.

(Dept. of Otolaryngology, The University of Melbourne, 550 Swanstone Street, Parkville Victoria 3010, Australia; e-mail:vogela@ unimelb.edu.au) Acoustic analysis of the effects of sustained wakefulness on speech. J. Acoust. Soc. Am. 128 (2010) 3747 